\title{
Reconstruction from Periodic Nonstationary Sampling (PNS) without Resampling
}

\author{
Bernard Lacaze \\ TeSA Lab., 7 Boulevard de la Gare \\ Toulouse, 31500, France \\ bernard.lacaze@tesa.prd.fr
}

\begin{abstract}
Periodic Nonuniform Samplings (PNS) are concatenations of periodic samplings of same periods but which are shifted. They allow to suppress aliasings, dead times of samplers by devices which provide a resampling of data. The obtained periodic sequence is treated through usual methods. In this paper, we show that the resampling stage is unnecessary, that PNS allow good estimations without resampling, at the same time for the sampled process, for linear filterings and for power spectra.
\end{abstract}

Key words and phrases : stationary processes, periodic nonuniform sampling, irregular sampling, resampling, power spectra.

2010 AMS Mathematics Subject Classification - 60G10, 60G35, 62D05, $94 \mathrm{~A} 20$

\section{Introduction}

1.1. The baseband case. In the time-continuous random process framework, sampling relates to a finite or denumerable set of real numbers (the sampling times) and the corresponding values of the process (the data). The challenge is to rebuild or to get close to the entire process from the set of data. The simplest situation deals with stationary processes and periodic sampling.

Let us consider a real or complex stationary process $\mathbf{Z}=\{Z(t), t \in \mathbb{R}\}$, with regular power spectral density $s_{Z}(\omega)$ defined by $[1]$

$$
\mathrm{E}\left[Z(t) Z^{*}(t-\tau)\right]=\int_{\Delta} e^{i \omega \tau} s_{Z}(\omega) d \omega
$$

where E[..] and the superscript * stand for the mathemetical expectation (the "ensemble mean") and the complex conjugate. The set $\Delta$ contains the spectral support of $\mathbf{Z}$. If $\mathbf{t}=\left\{t_{n}, n \in \mathbb{Z}\right\}$ is the set of sampling times, we look for reconstruction formulas

$$
Z(t)=\sum_{n \in \mathbb{Z}} a_{n}(t) Z\left(t_{n}\right) .
$$

In the periodic sampling case, $\mathbf{Z}^{\prime}=\{Z(n T), n \in \mathbb{Z}\}$ is the set of data, where $T$ is the sampling period. We know that an errorless (linear) reconstruction from $\mathbf{Z}^{\prime}$ 
depends on the length $|\Delta|$ of $\Delta$ defined in (1). When $\Delta=(-b, b)$ (the process is "baseband" in signal terminology), the Nyquist condition $T \leq \pi / b$ leads to the usual formula (Shannon, Nyquist...)

$$
Z(t)=\sum_{n \in \mathbb{Z}} \operatorname{sinc} \pi\left(\frac{t}{T}-n\right) Z(n T)
$$

where $\operatorname{sinc} x=(\sin x) / x$. When the sampling sequence $\mathbf{t}$ is not too different from $T \mathbb{Z}$, a resampling is the usual practice [1]. For instance, the system

$$
Z\left(t_{k}\right)=\sum_{n=-N}^{N} \operatorname{sinc} \pi\left(\frac{t_{k}}{T}-n\right) \widetilde{Z}(n T),|k| \leq N
$$

allows to compute the $\widetilde{Z}(k T),|k| \leq N$, which are estimations of the $Z(k T)$, in the case of a Cramer system. They are replaced in (3) to estimate $Z(t)$. Other solutions exist, for instance based on Lagrange interpolation [2], [3].

1.2. The two-bands case. In many physical cases, the power spectral density $s_{Z}(\omega)$ vanishes nearby the origin $\omega=0$. Waves which propagate in atmosphere belong to this class. In the real case, the support of $s_{Z}(\omega)$ is divided in two symmetric intervals (Z is "two-bands") :

$$
\Delta=(-b-a,-a) \cup(a, a+b), a \geq 0, b>0 .
$$

The Nyquist condition $T \leq \pi /(a+b)$ is replaced by the more favourable Landau condition $T \leq \pi / b$, which allows lower $T$ [4]. The "Shannon formula" (2) becomes the "generalized sampling formula"

$$
\left\{\begin{array}{l}
Z(t)=\sum_{n \in \mathbb{Z}} c_{n}(t) Z(n T) \\
c_{n}(t)=\operatorname{sinc}\left[\frac{\pi}{2}\left(\frac{t}{T}-n\right)\right] \cos \left[(2 N+1) \frac{\pi t}{2 T}+(-1)^{N+1} \frac{\pi n}{2}\right]
\end{array}\right.
$$

provided that

$$
\exists q \in \mathbb{N} \text { such that }(a, a+b) \subset(q / 2 T,(q+1) / 2 T) .
$$

It is the "anti-aliasing" condition, which prevents the realization of efficient devices. When the sampling sequence $\mathbf{t}$ is not periodic but not too different from $T \mathbb{Z}$, a resampling can be made from (4).

1.3. Periodic Nonstationary Sampling plan (PNS). The PNSN addresses the sampling sequence

$$
\mathbf{t}=\left\{n T+\theta_{k}, n \in \mathbb{Z}, k=1,2, . ., N\right\} .
$$

t is the concatenation of the $N$ periodic sequences of same period $T$

$$
\mathbf{t}_{k}=\left\{n T+\theta_{k}, n \in \mathbb{Z}\right\}, k=1,2, . ., N .
$$

For different $k$, the $\mathbf{t}_{k}$ have the same period $T$ but different delays $\theta_{k}$. Reconstruction in baseband was performed in a classical paper by J. L. Yen [5], [6]. 
Otherwise, the Landau theorem works. Because $T$ is equal to $N$-times the mean interval between the sampling times, we have

$$
T \leq 2 \pi N /|\Delta| .
$$

It is well established that this sampling plan is able to prevent drawbacks of aliasing. For instance in the two-bands case, $N=2$, adding the condition $2\left(\theta_{1}-\theta_{2}\right) \notin T \mathbb{Z}$. PNS models the following situations among others.

1) In wireless communications, processes are two-bands. The PNS2 prevents aliasing problems encountered in periodic sampling. Other estimations can be obtained about the analytic signal, and more generally, about linear filtering.

2) Samplers are electronic components which cut signals to create samples. The main characteristics of samplers are the rise time, the fall time and the dead time, which rules the minimum interval between two samples. A cheap sampler produces a deadtime too large to generate high frequency samplings. It is possible to lower the price of devices by means of several samplers in parallel, and delayed alternately. Each sampler is modelled by a sequence like (7) and a set of samplers is equivalent to the $\operatorname{PNS} N$ (6). To obtain a periodic sequence, the $\theta_{k}$ have to verify the conditions $\theta_{k}=\theta+k T / N$ for some $\theta$. This last operation is commonly performed, it is expensive and can be avoided, staying in the PNS framework [7].

3) In a more general context, an irregular sampling $\mathbf{t}=\left\{t_{n}, n \in \mathbb{Z}\right\}$ addresses an increasing sequence with a finite mean interval $T_{0}$ defined as

$$
\lim _{|n| \rightarrow \infty} \frac{t_{n}}{n}=T_{0} .
$$

$T_{0}=T / N$ for PNSN (6), $T_{0}=T$ for the periodic case. For instance, a jitter is modelled as (see example 5)

$$
t_{n}=n T_{0}+\varepsilon_{n},\left|\varepsilon_{n}\right|<T_{0} / 2 .
$$

In many circumstances, only a finite number of data is available. We will see that a PNS is suitable to give good estimations together of the process (see examples 5 and 6 ) and of the power spectrum (see example 6).

In this paper, we consider processes $\mathbf{Z}$ with power spectra defined inside the union of $N$ intervals of length $2 \pi / T$. The Landau rate is $T_{0}=T / N$ which corresponds to the PNSN plan (6). We construct a linear system $N \mathrm{x} N$ which allows to reconstruct $\mathbf{Z}$. When $N$ is large enough, this procedure provides good estimates of power spectra (and without resampling and whatever possible aliasings).

Section 2 explains the basic algorithm, and Section 3 develops examples. Possible improvements for a better convergence are given Section 4, non-stationary processes sampling is studied in Section 5, and Appendices give basic properties of stationary processes. 


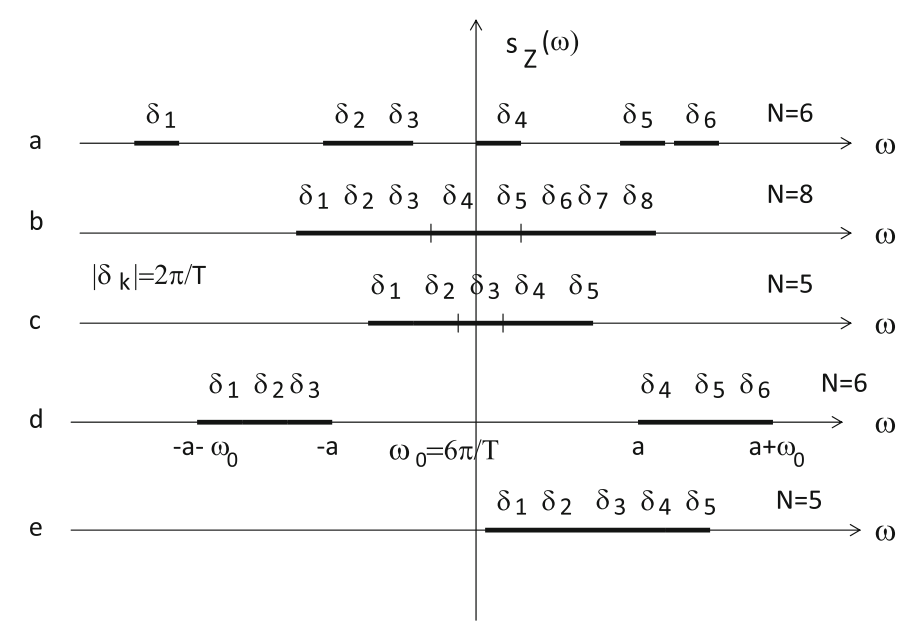

Figure 1. Some possible spectral supports. a: arbitrary - b: baseband, even $N$ - c: baseband, odd $N$ - d: two-bands, real process - e: analytic signal

\section{Reconstruction procedure}

We consider the set of $N$ intervals $\delta_{k}, k=1,2, . ., N$

$$
\delta_{k}=\left(a_{k}-\frac{\pi}{T}, a_{k}+\frac{\pi}{T}\right)
$$

with $a_{k+1} \geq a_{k}+\frac{2 \pi}{T}$. The set $\Delta=\cup_{k=1}^{N} \delta_{k}$ is (or contains) the spectral support of the stationary process $\mathbf{Z}=\{Z(t), t \in \mathbb{R}\}$.

Figure 1 depicts some examples (see Section 3). The process $\mathbf{Z}_{k}=\left\{Z_{k}(t), t \in \mathbb{R}\right\}$ is the output of the bandpass filter on $\delta_{k}$ with input $\mathbf{Z}$. This means that

$$
Z(t)=\sum_{k=1}^{N} Z_{k}(t)
$$

$\mathbf{Z}_{k}$ is the component of $\mathbf{Z}$ on $\delta_{k}$. Because $\left|\delta_{k}\right|=\frac{2 \pi}{T}$, each process $\mathbf{Z}_{k}$ can be reconstructed from the set of samples

$$
Z_{k}(n T+\theta), n \in \mathbb{Z}
$$

for any $\theta$. For instance (a variant of the classical sampling formula, see Appendix A)

$$
Z_{k}(t)=\sum_{n \in \mathbb{Z}} e^{i a_{k}(t-\theta-n T)} \operatorname{sinc}\left[\pi\left(\frac{t-\theta}{T}-n\right)\right] Z_{k}(n T+\theta) .
$$

Let assume that, for all $k, n$, it exists $d_{n}, b_{k}$ such that

$$
e^{-i a_{k} n T}=d_{n} / b_{k} \text {. }
$$

Holding (10) in (9), a summation leads to (we want eliminate the $Z_{k}(n T+\theta)$ which are not observed and we want replace them by the $Z(n T+\theta)$ which are 
the data, using (8))

$$
\sum_{k=1}^{N} b_{k} e^{-i a_{k}(t-\theta)} Z_{k}(t)=\sum_{n \in \mathbb{Z}} d_{n} \operatorname{sinc}\left[\pi\left(\frac{t-\theta}{T}-n\right)\right] Z(n T+\theta) .
$$

(10) is verified only when, for all $k=1, . ., N$

$$
a_{k} T=\alpha+2 \pi \mu_{k}, \alpha \in \mathbb{R}, \mu_{k} \in \mathbb{Z} .
$$

At this condition, (11) becomes

$$
\sum_{k=1}^{N} e^{-i a_{k}(t-\theta)} Z_{k}(t)=\sum_{n \in \mathbb{Z}} e^{-i n \alpha} \operatorname{sinc}\left[\pi\left(\frac{t-\theta}{T}-n\right)\right] Z(n T+\theta) .
$$

With $N$ distinct values $\theta_{l}$ of $\theta$, we obtain the following linear system with unknowns $U_{k}(t)=e^{-i a_{k} t} Z_{k}(t)$

$$
\left\{\begin{array}{c}
\mathbf{C}(t)=\mathbf{B U}(t) \\
\mathbf{B}=\left[e^{i a_{k} \theta_{l}}\right] \\
\mathbf{U}(t)=\left[e^{-i a_{k} t} Z_{k}(t)\right] \\
\mathbf{C}(t)=\left[\sum_{n \in \mathbb{Z}} e^{-i n \alpha} \operatorname{sinc}\left[\pi\left(\frac{t-\theta_{l}}{T}-n\right)\right] Z\left(n T+\theta_{l}\right)\right] .
\end{array}\right.
$$

$\mathbf{C}$ and $\mathbf{U}$ are column matrices filled by data (through the $Z\left(n T+\theta_{l}\right)$ ) and unknowns (through the $Z_{k}(t)$ ). B is a square matrix with $N^{2}$ elements and is independent of $t$, i.e $\mathbf{B}$ depends only on the sampling plan. If the $\theta_{l}$ are well-chosen, the matrix $\mathbf{B}$ is invertible and the system becomes

$$
\mathbf{U}(t)=\mathbf{B}^{-1} \mathbf{C}(t) .
$$

From (12), (14), (15), we find $Z_{k}(t)$ and $Z(t)$ from (8).

To summarize, we consider a random process $\mathbf{Z}$. Its power support $\Delta$ is divided in $N$ components $\delta_{k}$ of length $2 \pi / T(|\Delta|=2 \pi N / T)$. Provided that the determinant $|\mathbf{B}|$ of the matrix $\mathbf{B}=\left[e^{i a_{k} \theta_{l}}\right]$ is different from 0 , the PNSN

$$
\left\{n T+\theta_{k}, n \in \mathbb{Z}, k=1,2, . ., N\right\}
$$

of basic period $T$, is able to reconstruct $\mathbf{Z}$ without error when (12) is verified. (15) provides the unknowns $Z_{k}(t), Z(t)$ from the data $Z\left(n T+\theta_{l}\right)$ and without intermediary (i.e without resampling and without conditions coming from aliasing).

\section{Examples}

3.1. Example 1: baseband process. We assume that $\mathbf{Z}$ is a baseband process, i.e $\Delta=\left(-\omega_{0}, \omega_{0}\right) \cdot \Delta$ is divided in a even or odd number $N$ of adjacent intervals $\delta_{k}$ of length $2 \omega_{0} / N$. The first case $N=2 Q$ appears as limit in the following example (two-bands signals). So, we assume that $N=2 Q+1$ which successively leads to $T=\frac{2 \pi}{\left|\delta_{k}\right|}=\frac{\pi(2 Q+1)}{\omega_{0}}$ and

$$
\delta_{k}=\left(\omega_{0} \frac{2 k-2 Q-3}{2 Q+1}, \omega_{0} \frac{2 k-2 Q-1}{2 Q+1}\right), 1 \leq k \leq 2 Q+1
$$




$$
a_{k}=\omega_{0} \frac{2 k-2 Q-2}{2 Q+1}, 1 \leq k \leq 2 Q+1 .
$$

We obtain $e^{-i a_{k} n T}=1$ for all $k, n$. The conditions (10) or (12) are verified with, for instance $\alpha=0, b_{k}=d_{n}=1$ for all $k, n$. The basic equation (13) simplifies as

$$
\sum_{k=1}^{N} e^{-i a_{k}(t-\theta)} Z_{k}(t)=\sum_{n \in \mathbb{Z}} \operatorname{sinc}\left[\pi\left(\frac{t-\theta}{T}-n\right)\right] Z(n T+\theta) .
$$

As explained above, we can construct a Cramer system $N \mathrm{x} N$, given $N$ well chosen values $\theta_{1}, . ., \theta_{N}$ of the parameter $\theta$. The matrix $\mathbf{B}=\left[e^{i a_{k} \theta_{l}}\right]$ is of Vandermonde type, and will allow to reach the $Z_{k}(t)$ and $Z(t)$. This situation is treated in the functions framework in the founding papers [5], [6]. When $2 \pi / T$ is small and $N$ is large, the condition on $\omega_{0}\left(\omega_{0}\right.$ is a multiple of $\left.\pi / T\right)$ is unnecessary and the method allows not only a reconstruction but also an estimation of power spectra (see Section 3.6).

3.2. Example 2: two-bands processes. In communications, the most used shape for frequency bands is

$$
\Delta=\left(-\omega_{0}-a,-a\right) \cup\left(a, \omega_{0}+a\right)
$$

which returns to example 1 for $a=0$. When $N=2 Q$ and $T=\frac{2 \pi}{\left|\delta_{k}\right|}=\frac{2 \pi Q}{\omega_{0}}$

$$
\begin{gathered}
\delta_{k}=\left\{\begin{array}{c}
\left(-a+\frac{(k-Q-1) \omega_{0}}{Q},-a+\frac{(k-Q) \omega_{0}}{Q}\right), 1 \leq k \leq Q \\
\left(a+\frac{(k-Q-1) \omega_{0}}{Q}, a+\frac{(k-Q) \omega_{0}}{Q}\right), Q+1 \leq k \leq 2 Q
\end{array}\right. \\
a_{k}=\left\{\begin{array}{c}
-a+\frac{(2 k-2 Q-1) \omega_{0}}{2 Q}, 1 \leq k \leq Q \\
a+\frac{(2 k-2 Q-1) \omega_{0}}{2 Q}, Q+1 \leq k \leq 2 Q .
\end{array}\right.
\end{gathered}
$$

We obtain

$$
e^{-i a_{k} n T}=\left\{\begin{array}{c}
(-1)^{n} e^{2 i \pi n Q a / \omega_{0}}, 1 \leq k \leq Q \\
(-1)^{n} e^{-2 i \pi n Q a / \omega_{0}}, Q+1 \leq k \leq 2 Q
\end{array}\right.
$$

which does not verify conditions (10) or (12) for $a \neq 0$, except when

$$
\frac{2 Q a}{\omega_{0}} \in \mathbb{N}
$$

In real situations, it is possible to verify (17) from negligible modifications of $a$ and/or $\omega_{0}$. When it is the case, the following equation is verified

$$
\begin{gathered}
\sum_{k=1}^{N} e^{-i a_{k}(t-\theta)} Z_{k}(t)= \\
\sum_{n \in \mathbb{Z}} e^{i \pi n(\alpha+1)} \operatorname{sinc}\left[\pi\left(\frac{t-\theta}{T}-n\right)\right] Z(n T+\theta) .
\end{gathered}
$$

Example 5 illustrates a realistic extension of the method where the sampling sequence (i.e the $\theta$ ) varies with $t$. 
3.3. Example 3: the analytic signal. The analytic signal is a useful tool which defines envelopes and phases. The analytic signal $\mathbf{U}$ of the real process $\mathbf{X}$ is defined by

$$
U(t)=X(t)+i \int_{-\infty}^{\infty} \frac{X(u)}{\pi(t-u)} d u
$$

where the integral (defined in the Cauchy sense) is the Hilbert transform of $\mathbf{X}$ [1]. We know that $\mathbf{U}$ is two times the component of $\mathbf{X}$ with positive frequencies. Equivalently, in the context of Example 2, for $N=2 Q$

$$
U(t)=2 \sum_{k=Q+1}^{2 Q} X_{k}(t)
$$

which can be calculated from (18) taking $N=2 Q$ well-chosen values of $\theta$. The problem is not so simple when $N=2 Q+1$, where

$$
U(t)=2 X_{Q+1}^{+}+2 \sum_{k=Q+2}^{2 Q+1} X_{k}(t) .
$$

$\mathbf{X}_{Q+1}^{+}$is the part of $\mathbf{X}_{Q+1}$ with positive frequencies. Among other solutions, we have $\mathbf{X}_{Q+1}^{+}=\mathbf{Y}_{Q+1}$ where (see Example 4 below)

$$
\mathbf{Y}=\mathcal{F}[\mathbf{X}], F(\omega)=F\left(\omega+\frac{2 \pi}{T}\right)=\left\{\begin{array}{c}
1, \omega \in(0, \pi / T) \\
0, \omega \in(-\pi / T, 0)
\end{array}\right.
$$

3.4. Example 4: filtering. 1) Let assume that, for all $k$

$$
a_{k} \in \frac{2 \pi}{T} \mathbb{Z}
$$

and let consider a linear invariant filter $(\mathrm{LIF}) \mathcal{F}$ with complex gain $F(\omega)$, some periodic function with period $2 \pi / T$. This means that (see Appendix B)

$$
\mathcal{F}\left[\boldsymbol{Z}_{k}\right](t)=e^{i t a_{k}} \sum_{n=-\infty}^{\infty} f_{n}(t) Z_{k}(n T), f_{n}(t)=\frac{T}{2 \pi} \int_{-\pi / T}^{\pi / T} F(\omega) e^{i \omega(t-n T)} d \omega .
$$

Consequently, for all $\theta$

$$
\sum_{k=1}^{N} \mathcal{F}\left[\boldsymbol{Z}_{k}\right](t) e^{-i(t-\theta) a_{k}}=\sum_{n=-\infty}^{\infty} f_{n}(t-\theta) Z(n T+\theta) .
$$

Giving $N$ well-chosen values $\theta_{j}$ of $\theta,(21)$ leads to a linear $N \mathrm{x} N$ system which allows to compute the $\mathcal{F}\left[\boldsymbol{Z}_{k}\right](t)$ in function of the $Z\left(n T+\theta_{j}\right)$ and then $\mathcal{F}[\boldsymbol{Z}](t)$ from

$$
\mathcal{F}[\boldsymbol{Z}](t)=\sum_{k=1}^{N} \mathcal{F}\left[\boldsymbol{Z}_{k}\right](t) .
$$

2) If, for all $k$

$$
a_{k} \in \frac{\pi}{T}(2 \mathbb{Z}+1)
$$


the results above become

$$
\begin{gathered}
\sum_{k=1}^{N} \mathcal{F}\left[\boldsymbol{Z}_{k}\right](t) e^{-i(t-\theta) a_{k}}=\sum_{n=-\infty}^{\infty}(-1)^{n} f_{n}(t-\theta) Z(n T+\theta) \\
f_{n}(t)=\frac{(-1)^{n} T}{2 \pi} \int_{-\pi / T}^{\pi / T} F\left(\omega+\frac{\pi}{T}\right) e^{i \omega(t-n T)} d \omega .
\end{gathered}
$$

Section 4 will take into account these results to improve convergence rates of reconstruction formulas.

3.5. Example 5: irregular sampling and reconstruction. The PNSN is a first step in the direction of irregular sampling treatment. Let consider an increasing sequence of real numbers $\left\{t_{n}, n \in \mathbb{Z}\right\}$, only subject to conditions at the infinite as

$$
\lim _{|n| \rightarrow \infty} \frac{t_{n}}{n}=\mu>0, \lim _{n \rightarrow \infty}\left[\frac{1}{t_{n}}+\frac{1}{t_{-n}}\right]=\mu^{\prime}
$$

where $\mu$ and $\mu^{\prime}$ are finite. In the case of a PNSN of basic period $T$, we have $\mu=T / N, \mu^{\prime}=0$. The "Lagrange estimator" $\widetilde{Z}(t)$ could be defined from limits of Lagrange polynomials, elsewhere extensively covered in the literature of analytic functions [8], [9]

$$
\widetilde{Z}(t)=\sum_{n \in \mathbb{Z}}\left[\prod_{m \neq n} \frac{t-t_{m}}{t_{n}-t_{m}}\right] Z\left(t_{n}\right) .
$$

Unfortunately, we are not sure that (26) is finite, and if it is the case, a nonmeasurable and unpredictable error will appear because, in reality, only a finite number of $t_{n}$ is known close to a chosen value of $t$. Also, the mathematical theory is written in the Nyquist framework, i.e for functions in baseband (with Fourier transform which cancels outside an interval as $\left.\left(-\omega_{0}, \omega_{0}\right)\right)$. When conditions $(25)$ are fulfilled, it is possible to construct sequences of PNS $N$ which converge when $N$ increases indefinitely [2], [3].

In the Landau context, the problem remains unsolved (to my knowledge). Let assume that we observe the process $\mathbf{Z}$ at $N$ times $t_{1}, . ., t_{N}$ around $t$, with large enough $N$. It is reasonable to think that the PNS $N$, with $T$ in accordance with the Landau condition, will provide a good estimation $\widetilde{Z}(t)$ of $Z(t)$ (knowing the spectral support of $\mathbf{Z}$ ). Unfortunately, a mathematical proof is conditional on the matrix $\mathbf{B}^{-1}$ convergence for large values of $N$. This study is beyond my expertise.

Figure 2 illustrates the reconstruction of a two-bands process when the sampling is submitted to a random (but observed) jitter [10]. The process and its reconstruction are undistinguishable, unless strong enlargement. $\mathbf{Z}$ is a real process which is made of a Gaussian noise in baseband with spectral support of width $\pi$ and modulated in the bands $(-5 \pi,-4 \pi) \cup(4 \pi, 5 \pi)$ (i.e multiplied by $\cos (9 \pi t / 2))$. The Landau rate is equal to 1 and the Nyquist one is equal to 5 . Moreover, we have taken $N=20$, so that the estimation of $Z(t)$ is based on 10 samples before $t$ and 10 after $t$. The sequence of sampling times slides with $t$, and the PSN20 is based on elements surrounding $t$. We have simultaneously

$$
t_{k(t)} \leq t<t_{k(t)+1}, \theta_{j+10}=t_{j+k(t)},-9 \leq j \leq 10 .
$$




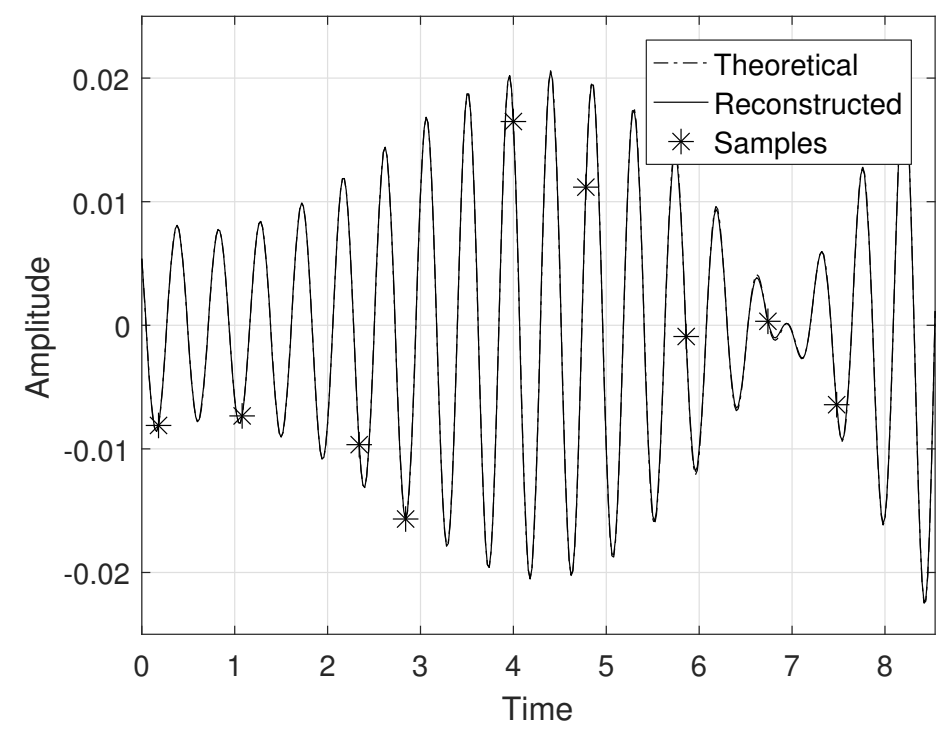

Figure 2. Two-bands processes. Periodic sampling with jitter. The theoretical and reconstructed processes are superimposed

Results depicted figure 2 show that the value $N=20$ is sufficient for very good results. We verify that the mean space between sampling times is slighty smaller than the unit (the Landau rate), and not $1 / 5$, as imposed in the Nyquist framework.

3.6. Example 6: irregular sampling and power spectra. PNSN allow to suppress drawbacks due to aliasings and dead times. Another application is the measure of power spectra. In this example, $N$ is the number of known sampling times $\left(t_{1}, t_{2}, . ., t_{N}\right)$, and $N$ determines $T$ knowing $|\Delta|$, the length of the spectral support. $Z\left(t_{k}\right)$ is given, but $Z\left(t_{k}+n T\right), n \neq 0$, is unknown. The error due to the lack of data is negligible when the distance between observed data and others is large enough.

The Vostok ice core was extracted in Antartica above a lake covered by around four kilometres ice thickness. The cutting up of the core and the chemical study of pieces allow an estimation of variations in time of temperature, of $\mathrm{CO}_{2}$ (carbon dioxide), $\mathrm{CH}_{4}$ (methane)... concentrations, at irregular times which are function of annual snowfall thickness. Results give a view of climate in the last 420000 years [11].

In the case of $\mathrm{CO}_{2}$ concentration, we have $N=282$ measures at times $t_{1}=$ $0, t_{2}, \ldots, t_{N}=l=420000$ (around one measure each 1500 years and we omit one data point to work with an even number). Consistent studies agree with a power spectrum in the frequential interval $\left(-7.10^{-5}, 7.10^{-5}\right)\left(\right.$ in year $\left.^{-1}\right)$ [13]. If we take as model a PNS $N$ with $\theta_{k}=t_{k}, k=1, \ldots, 282$, the Nyquist bound (which, in baseband, is the Landau bound) leads to 


$$
T=282 / 14.10^{-5} \approx 2.10^{6} \mathrm{y}
$$

$(\mathrm{y}=$ year $)$. In the model, the sampling times are

$$
t_{k}+n T, k=1, . ., 282, n \in \mathbb{Z} .
$$

For $t \in(0,420000)$, the near sampling times correspond to $n=0$, and otherwise the nearest correspond to $n= \pm 1$, at a distance at least equal to $16.10^{5}$ years. It is reasonable to discard them (and we do not have a choice). Then, the formula (13) is reduced to

$$
\sum_{k=1}^{N} e^{-i a_{k}\left(t-t_{j}\right)} \widetilde{Z}_{k}(t)=\operatorname{sinc}\left[\pi\left(\frac{t-t_{j}}{T}\right)\right] Z\left(t_{j}\right), j=1, . ., N .
$$

The result of the estimation is depicted in figure 3. We have to keep in mind that measurement errors are due to errors on concentration values and on sampling times, because the ice date is not the gases date [14]. This is why the estimation from a large number of data (here 282) seems better that from few neighbours (as for splines). Figure 3 gives an estimation of the near future, from a past which does not take into account the anthropogenic imprint.

In this example, we have to treat a linear system with 282 unknowns. Each of them $Z_{k}(t)$ occupates a spectral width equal to (in frequency)

$$
\frac{\left|\delta_{k}\right|}{2 \pi}=\frac{14.10^{-5}}{2 \pi \cdot 282}=8.10^{-8} \mathrm{y}^{-1}
$$

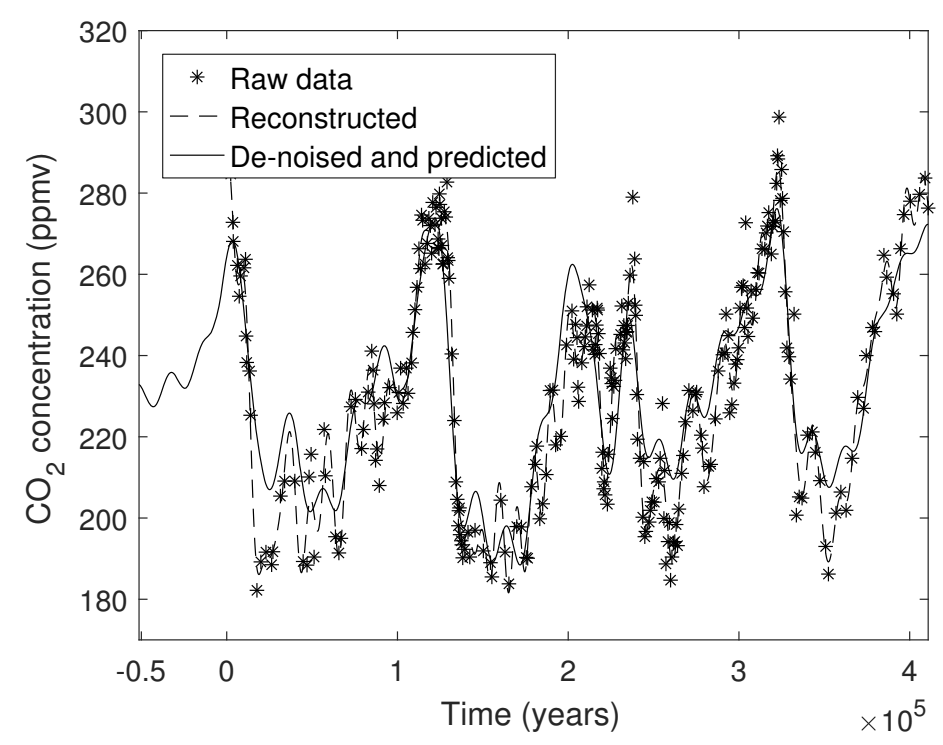

Figure 3. Vostok ice core. Reconstruction (dashed), data (stars), denoised and predicted (continuous line) for $\mathrm{CO}_{2}$ from the Vostok ice core 


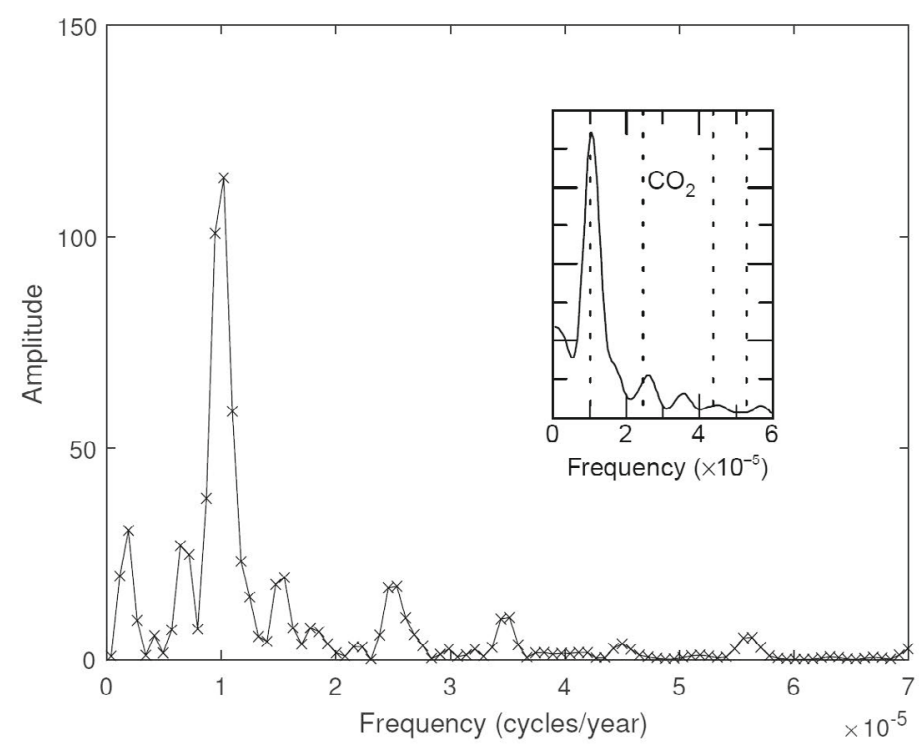

Figure 4. Power Spectral Density for $\mathrm{CO}_{2}$ from the Vostok ice core. Each cross denotes power in a calibrated interval. The incrustation illustrates original result in [11]

with respect to $|\Delta| / 2 \pi=14.10^{-5} \mathrm{y}^{-1}$. Specialists are interested in spectral lines, i.e thin places which hold strong powers with respect to surrounding places. Equivalently, we look for indices $k$ with extraordinary values of $\mathrm{P}_{k}=\mathrm{E}\left[\left|Z_{k}(t)\right|^{2}\right]$. For these values, $Z_{k}(t)$ is close to a monochromatic wave, and because the $\delta_{k}$ are small enough, the set of $\mathrm{P}_{k}$ provides a good approximation of the spectral density.

Figures 3, 4 and 5 illustrate results about $\mathrm{CO}_{2}$ data [11], which are widely studied in [12]. Figure 3 depicts the reconstruction (dashed line), the data (stars) and the denoised and predicted evolution (continuous line), independent of anthropogenic pollution.

Figure 4 shows the power spectral density (actually the power in $\delta_{k}$ ) obtained using (22). Concentrations of power appear for values of $k$ corresponding to frequencies (in $10^{-5} \mathrm{y}^{-1}$ ) around $0.25,0.75,1,1.5,2.5,3.5,4.5,5.5$. The insert is the result shown in [11]. Improvements with respect to [11] can be found in [13], and comparisons have to be made with results of this paper.

Figure 5 depicts the main line evolution at $10^{-5} \mathrm{y}^{-1}$ (continuous line), and its extrapolation outside the interval of data. It represents more than $40 \%$ of the total power. Comparisons with Milankovitch cycles remains a disputed question [15].

To summarize, given $N$ data at irregular dates, near enough with respect to the power spectral support length, a PNSN is able to provide formulas of reconstruction, not only matched to the whole process, but giving bandpass 


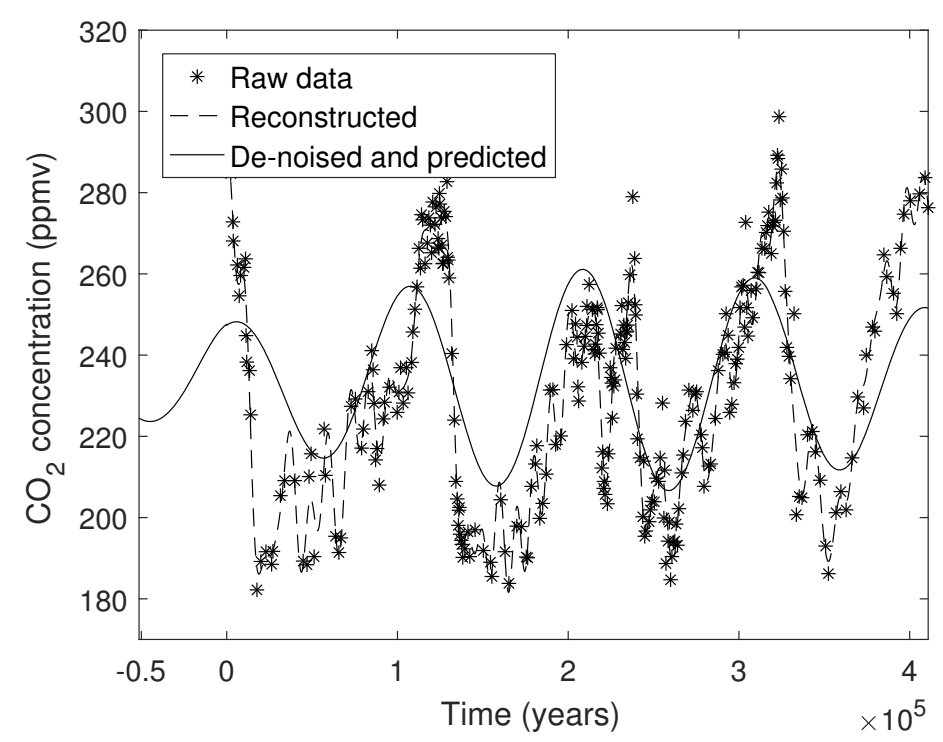

Figure 5. Vostok ice core. $\mathrm{CO}_{2}$ concentration extraction of the main spectral component at $10^{-5} \mathrm{y}^{-1}$ (more than $40 \%$ of the total power)

filterings, and power spectral estimations which are better than those proposed in literature. Even in baseband, this method seems to give more possibilities than others based on modifications of the Lagrange interpolation formula [2].

3.7. Example 7: cases $N=2$ and $N=3$. Formulas obtained above are true whatever the order $N$ of the PNS. They imply the inversion of matrices of $N \mathrm{x} N$ size. For instance, we have $N=282$ for the Vostok ice core detailed in example 6 . Today, computers do not have difficulties with this problem. Though condition (12) is not very constraining, particularly when $T$ is large enough, it is the condition which allows to justify (14). The question of knowing if it is a necessary condition of errorless reconstruction is a good one (the answer is no).

In cases $N=2$ or 3 , it is possible to determine the best mean-square estimation. Its standard deviation (the error) will give necessary conditions of errorless reconstruction. Though the method could be applied for any $N$, the computations become too tedious already for $N=4$. The best mean-square estimator $\widetilde{Z}(t)$ of $Z(t)$ is the orthogonal projection on the Hilbert space of data. The result is a circuit of LIF [16], [17]. The complete study of the PNS2 for instance shows that the method developed in Section 2, though powerful, is not optimal [18]. Let assume that $N=2, T=1, \theta_{1}=0, \theta_{2}=\theta$ to simplify notations. The study of the "generalized folded spectrum" $S_{u}(\omega)$ is the key of the problem. We define it by

$$
S_{u}(\omega)=\sum_{n \in \mathbb{Z}} e^{2 i \pi n u} s_{Z}(\omega+2 n \pi) .
$$




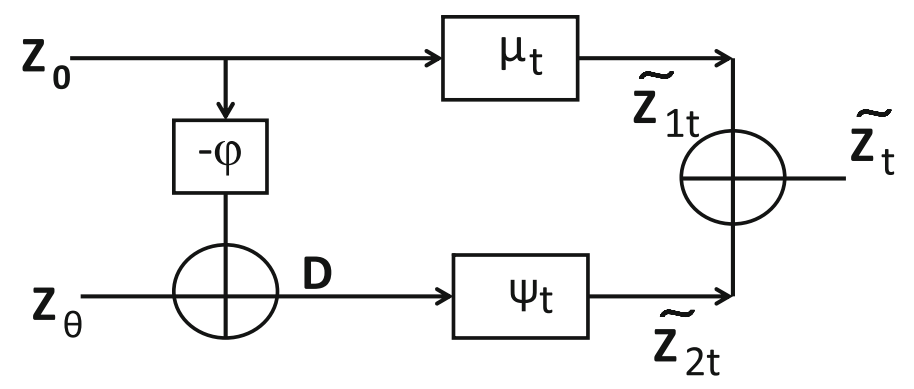

Figure 6. Optimal circuit for PNS2, defined by 3 LIF [16], [18]

The best mean-square estimation $\widetilde{Z}(t)$ of $Z(t)$ is the orthogonal projection

$$
\widetilde{Z}(t)=\operatorname{pr}_{\mathbf{H}_{0}+\mathbf{H}_{\theta}} Z(t)
$$

where $\mathbf{H}_{u}$ is the Hilbert space spanned by the stationary sequence

$$
\mathbf{Z}_{u}=\{Z(n+u), n \in \mathbb{Z}\}
$$

i.e the set of random variables linearly constructed from the $Z(n+u)$, for some $u$. The difficulty comes from the fact that both Hilbert spaces $\mathbf{H}_{0}$ and $\mathbf{H}_{\theta}$ are not orthogonal most of the time. When we have

$$
\mathbf{H}_{0}+\mathbf{H}_{\theta}=\mathbf{H}_{0}+\mathbf{K}_{\mathbf{D}}, \mathbf{H}_{0} \perp \mathbf{K}_{\mathbf{D}}
$$

we also have $\left(\mathbf{K}_{\mathbf{D}}\right.$ is the Hilbert space spanned by some stationary sequence $\left.\mathbf{D}=\left\{D_{n}, n \in \mathbb{Z}\right\}\right)$

$$
\widetilde{Z}(t)=\operatorname{pr}_{\mathbf{H}_{0}} Z(t)+\operatorname{pr}_{\mathbf{K}_{\mathbf{D}}} Z(t)=\widetilde{Z}_{1}(t)+\widetilde{Z}_{2}(t) .
$$

Figure 6 depicts a pattern allowing to obtain $\widetilde{Z}(t)$ from three (numerical) LIF, of complex gains $\phi, \mu_{t}, \psi_{t}$, which define the sequence $\mathbf{D}$ and the sequences

$$
\widetilde{\mathbf{Z}}_{1 t}=\left\{\widetilde{Z}_{1}(t+n), n \in \mathbb{Z}\right\}, \widetilde{\mathbf{Z}}_{2 t}=\left\{\widetilde{Z}_{2}(t+n), n \in \mathbb{Z}\right\}
$$

of projections on $\mathbf{H}_{0}$, and on $\mathbf{K}_{\mathbf{D}}$. Some algebra leads to

$$
\begin{gathered}
\sigma_{t}^{2}=\mathrm{E}\left[|Z(t)-\widetilde{Z}(t)|^{2}\right]= \\
\int_{-\pi}^{\pi}\left[\frac{S_{0}^{2}-\left|S_{t}\right|^{2}}{S_{0}}-\frac{\left|S_{0} S_{t-\theta}-S_{t} S_{\theta}^{*}\right|^{2}}{S_{0}\left(S_{0}^{2}-\left|S_{\theta}\right|^{2}\right)}\right](\omega) d \omega .
\end{gathered}
$$

Let define the sets $\Delta_{n} \subset(-\pi, \pi)$ of $\omega$ such that exactly $n$ integers $k_{1}, k_{2}, \ldots, k_{n}$ verify

$$
s_{Z}\left(\omega+2 k_{l} \pi\right)>0 .
$$

It is not difficult to see that $\sigma_{t}=0$ for all $t$ when

$$
(-\pi, \pi)=\Delta_{0} \cup \Delta_{1} \cup \Delta_{2} .
$$




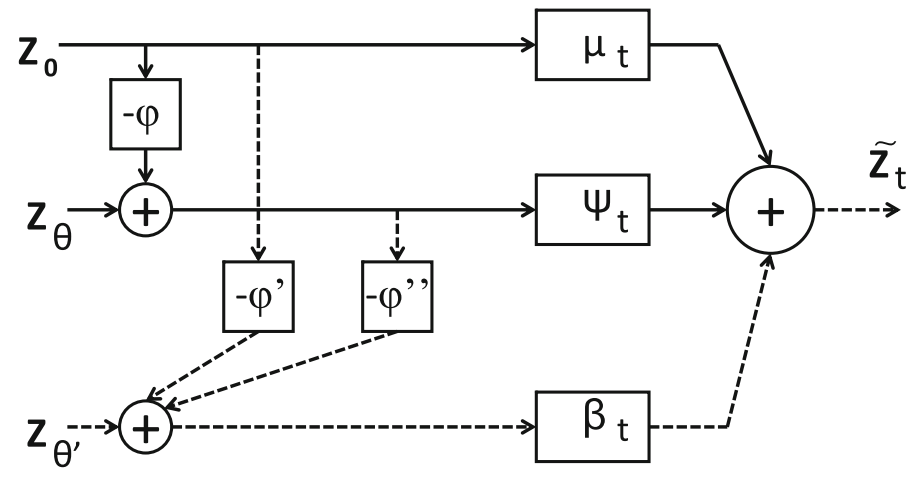

Figure 7. PNS3 circuit for least mean square reconstructions [17]. In continuous lines, circuit for PNS2 (figure 6)

This means that the PNS2 overcomes "one spectral folding". It is the case when the power is distributed on two intervals of width $2 \pi$, whatever their bounds. This implies that an errorless reconstruction is possible in Example 2, Section 3.2 , even if the condition (17) is not fulfilled.

The case $N=3$ is illustrated by figure 7 . It is ruled by a supplementary line with respect to figure 6 , and three new (numerical) LIF of complex gains $\phi^{\prime}, \phi^{\prime \prime}$ and $\beta_{t}$, with two of them which link the new line with the the other. This scheme can overcome "two spectral foldings", and so on when $N$ increases. The scheme is recursive, because the complex gains $\phi, \mu_{t}, \psi_{t}$ are unchanged, and the property remains for $\operatorname{PNS} N, N>3$, though computations become more laborious.

The Landau condition is necessary [4], but it is always an approximation in the real world. It is this flexibility which allows to fulfill (12), playing with bounds of intervals containing power.

\section{Convergence improvements}

By construction from the usual sampling formula, series (9) converge in $1 / n$. The problem comes from the discontinuity of $e^{i \omega t}$ at the bounds of the interval of $\omega$ where this function is expanded in Fourier series (except for particular values of $t$ and modulo $2 \pi / T$ ). But it is possible to suppress this drawback.

1) To simplify, we put ourselves in baseband, assuming that the Nyquist condition is verified for both $N=2 Q$ and $N=2 Q+1$. This means some oversampling, due to the condition $s_{Z}(\omega)=0,|\omega|>\frac{2 \pi}{T} Q$. In the first case (see Sections 3.1 and 3.2)

$$
\delta_{k}=\left((k-1-Q) \frac{2 \pi}{T},(k-Q) \frac{2 \pi}{T}\right), 1 \leq k \leq 2 Q
$$

In the other case (we note $\delta_{k}^{\prime}, \mathbf{Z}_{k}^{\prime}$ instead of $\delta_{k}, \mathbf{Z}_{k}$ )

$$
\delta_{k}^{\prime}=\left((2 k-3-2 Q) \frac{\pi}{T},(2 k-1-2 Q) \frac{\pi}{T}\right), 1 \leq k \leq 2 Q+1 .
$$




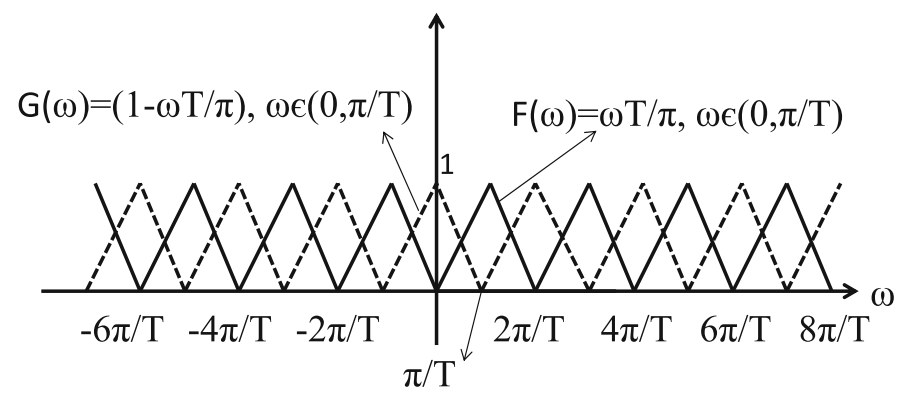

Figure 8. $F(\omega)$ and $G(\omega)$ are complementary filters complex gains, which improves the convergence of reconstruction formulas

We define the LIF $\mathcal{F}$ and $\mathcal{G}$ by their complex gains $F(\omega), G(\omega)$ periodic with period $2 \pi / T$ (see Appendix B)

$$
F(\omega)=F(-\omega)=G\left(\omega-\frac{\pi}{T}\right)=\frac{\omega T}{\pi}, \omega \in\left(0, \frac{\pi}{T}\right) .
$$

Using Example 4 and particularly (22), it is clear that $\left(\mathbf{Z}_{k}^{\prime}\right.$ is the part of $\mathbf{Z}$ on $\left.\delta_{k}^{\prime}\right)$

$$
Z(t)=\sum_{k=1}^{2 Q} \mathcal{F}\left[\mathbf{Z}_{k}\right](t)+\sum_{k=1}^{2 Q+1} \mathcal{G}\left[\mathbf{Z}_{k}^{\prime}\right](t)
$$

because, for all $\omega$ (see figure 8 )

$$
F(\omega)+G(\omega)=1 .
$$

The expansions of $F(\omega) e^{i \omega t}$ and $G(\omega) e^{i \omega t}$ on $\delta_{k}$ and $\delta_{k}^{\prime}$ are respectively

$$
\begin{gathered}
F(\omega) e^{i \omega t}=\sum_{n \in \mathbb{Z}} \frac{(-1)^{n}}{2} e^{i \pi t(2 k-1-2 Q) / T} \operatorname{sinc}^{2}\left[\frac{\pi}{2}\left(\frac{t}{T}-n\right)\right] e^{i n \omega T}, \omega \in \delta_{k} \\
G(\omega) e^{i \omega t}=\frac{1}{2} e^{i \pi t(2 k-2-2 Q) / T} \operatorname{sinc}^{2}\left[\frac{\pi}{2}\left(\frac{t}{T}-n\right)\right] e^{i n \omega T}, \omega \in \delta_{k}^{\prime} .
\end{gathered}
$$

Equivalently,

$$
\begin{aligned}
& \mathcal{F} {\left[\mathbf{Z}_{k}\right](t) e^{-i \pi(t-\theta)(2 k-1-2 Q) / T}=\frac{1}{2} \sum_{n \in \mathbb{Z}}(-1)^{n} \operatorname{sinc}^{2}\left[\frac{\pi}{2}\left(\frac{t-\theta}{T}-n\right)\right] Z_{k}(n T+\theta) } \\
& \mathcal{G}\left[\mathbf{Z}_{k}^{\prime}\right](t) e^{-i \pi(t-\theta)(2 k-2-2 Q) / T}=\frac{1}{2} \sum_{n \in \mathbb{Z}} \operatorname{sinc}^{2}\left[\frac{\pi}{2}\left(\frac{t-\theta}{T}-n\right)\right] Z_{k}^{\prime}(n T+\theta) .
\end{aligned}
$$

A summation on $k$ allows to introduce the $Z(n T+\theta)$ (instead of the $Z_{k}(n T+\theta)$ and the $\left.Z_{k}^{\prime}(n T+\theta)\right)$. With at most $4 Q+1$ values of $\theta$, we obtain two linear system providing the $\mathcal{F}\left[\mathbf{Z}_{k}\right](t)$ and the $\mathcal{G}\left[\mathbf{Z}_{k}^{\prime}\right](t)$ and then $Z(t)$. Because terms in sinc ${ }^{2}$ have replaced terms in sinc, the convergence of formulas is now in $1 / n^{2}$.

2 ) This result comes from (22) and from the conditions at the bounds of $\delta_{k}$ (for $F(\omega)$ ) and of $\delta_{k}^{\prime}$ (for $G(\omega)$ ) which imply the continuity of $F(\omega) e^{i \omega t}$ and $G(\omega) e^{i \omega t}$ because $\left.F(0)=G(\pi / T)=0\right)$. It is easy to find more general solutions. 
Let $F(\omega)$ be periodic with period $2 \pi / T$ and

$$
F(\omega)=\sum_{n \in \mathbb{Z}} a_{n} e^{i n \omega T} .
$$

We have the equivalences (provided that the sum above is well defined)

$$
\begin{gathered}
F(\omega)+F\left(\omega+\frac{\pi}{T}\right)=1 \\
F(0)=F\left(\frac{2 \pi}{T}\right)=0
\end{gathered} \Longleftrightarrow \begin{gathered}
a_{0}=1, a_{2 n}=0, n \neq 0 \\
\sum_{n \in \mathbb{Z}} a_{2 n+1}=-\frac{1}{2} .
\end{gathered}
$$

For instance, the function $F(\omega)=\frac{1}{2}\left(1-e^{i \omega T}\right)$ fulfills these conditions, and (29) is replaced by

$$
\begin{gathered}
\mathcal{F}\left[\mathbf{Z}_{k}\right](t) e^{-i \pi(t-\theta)(2 k-1-2 Q) / T}=\frac{1}{2 \pi} \sum_{n \in \mathbb{Z}} \frac{\sin \frac{\pi}{T}(t-\theta)}{\left(\frac{t-\theta}{T}-n\right)\left(\frac{t-\theta}{T}-n+1\right)} Z_{k}(n T+\theta) \\
\mathcal{G}\left[\mathbf{Z}_{k}^{\prime}\right](t) e^{-i \pi(t-\theta)(2 k-2-2 Q) / T}=\frac{(-1)^{n}}{2 \pi} \sum_{n \in \mathbb{Z}} \frac{\sin \frac{\pi}{T}(t-\theta)}{\left(\frac{t-\theta}{T}-n\right)\left(\frac{t-\theta}{T}-n+1\right)} Z_{k}^{\prime}(n T+\theta)
\end{gathered}
$$

which decrease in $1 / n^{2}$ as expected.

3) Better convergence rates are obtained, adding conditions of regularity about $F(\omega)$. The supplementary condition

$$
F^{\prime}(0)=F^{\prime}\left(\frac{2 \pi}{T}\right)=0 \Longrightarrow \sum_{n \in \mathbb{Z}} n a_{2 n+1}=\frac{1}{4}
$$

leads to a convergence in $1 / n^{3}$. For instance, the following functions fulfill these conditions

$$
F(\omega)=\frac{1}{4}\left(2-3 e^{i \omega T}+e^{3 i \omega T}\right), F(\omega)=\frac{1}{2}(1-\cos \omega T)
$$

and, for the second one, (31), (32) become

$$
\begin{gathered}
\mathcal{F}\left[\mathbf{Z}_{k}\right](t) e^{-i \pi(t-\theta)(2 k-1-2 Q) / T}= \\
\frac{-1}{2 \pi} \sum_{n \in \mathbb{Z}} \frac{\sin \frac{\pi}{T}(t-\theta)}{\left(\frac{t-\theta}{T}-n\right)\left(\frac{t-\theta}{T}-n+1\right)\left(\frac{t-\theta}{T}-n-1\right)} Z_{k}(n T+\theta) \\
\mathcal{G}\left[\mathbf{Z}_{k}^{\prime}\right](t) e^{-2 i \pi(t-\theta)(k-1-Q) / T}= \\
\frac{1}{2 \pi} \sum_{n \in \mathbb{Z}} \frac{(-1)^{n+1} \sin \frac{\pi}{T}(t-\theta)}{\left(\frac{t-\theta}{T}-n\right)\left(\frac{t-\theta}{T}-n+1\right)\left(\frac{t-\theta}{T}-n-1\right)} Z_{k}^{\prime}(n T+\theta) .
\end{gathered}
$$

Summations on $k$ in (33), (34), introduce the data $Z(n T+\theta)$ for good values of $\theta$, and tractable linear systems. The convergence rate can be increased up to $1 / n^{l}, l>3$, adding new but simple conditions.

\section{Sampling and nonstationarity}

In this paper, the stationarity of processes is characterized by a property of autocorrelation functions expressed by (1) for some $\Delta$ (when hypotheses of the 
Bochner-Khinchine are fulfilled [1]). For a random sequence $\mathbf{U}^{\prime}=\{U(n), n \in \mathbb{Z}\}$, this stationarity condition is replaced by (Herglotz lemma [19])

$$
\mathrm{E}\left[U(n) U^{*}(n-m)\right]=\int_{\Delta} e^{i \omega m} s_{U}(\omega) d \omega
$$

for $n, m \in \mathbb{Z}, \Delta \subset(-\pi, \pi)$.

The generic name "Periodic Nonstationary Sampling" for the sequence (6) is unusual. We understand the part "periodic" which matchs subsequences (7). The term "nonstationary" could be attributed either to the sequence $\mathbf{t}$ of (6) or to the samples (the $\left.Z\left(n T+\theta_{k}\right)\right)$. The periodic case $\left(\theta_{k}=k T / N\right)$ leads to a stationary sequence of data. For another set of $\theta_{k}$, the sequence of data is no longer stationary. I think that it is the good explanation. Moreover, it is clear that the set of $\theta_{k}$ has to be known if we want errorless estimations, and problems of estimations become hard when a random character is put on the $\theta_{k}$.

Previous sections are done for stationary processes with particular power spectra and matched PNS. To extend the field of applications to nonstationary processes, we can consider the class of harmonizable processes where the notion of power spectrum is available [19], [20]. $\mathbf{Z}$ is harmonizable when

$$
\mathrm{E}\left[Z(t) Z^{*}\left(t^{\prime}\right)\right]=\iint_{\Delta} e^{i u t-i v t^{\prime}} d S_{Z}(u, v)
$$

with, for instance, $\mathrm{E}\left[|Z(t)|^{2}\right] \leq c<\infty . S_{Z}(u, v)$ defines the two-dimensional power spectrum, and $\Delta$ one of its "supports". We will not develop the mathematical meaning of this two-dimensional Fourier transform. The set $\Delta$ has to verify consequences of the Schwarz inequality and the Hermitian symmetry [19], [21]. Let's give some examples.

1) $U(t)=2 A$ sinct, where $A$ is Gaussian $\mathrm{N}(0,1)$. $\mathbf{U}$ is harmonizable with

$$
\Delta=(-1,1)^{2}, s_{U}(u, v)=\frac{\delta^{2} S_{U}}{\delta u \delta v}(u, v)=1 .
$$

2) The notion of linear invariant filter is available for harmonizable processes (see Appendix B). If $F(\omega)$ is the spectral gain of the LIF $\mathcal{F}$, and if $\mathbf{U}$ is harmonizable, the power spectrum of $\mathbf{Z}=\mathcal{F}[\mathbf{U}]$ verifies (when $S_{U}$ is regular enough)

$$
s_{Z}(u, v)=F(u) F^{*}(v) s_{U}(u, v) .
$$

If $F(u)=1, u \in(a, b) \cup(-b,-a), 0 \leq a<b$, and 0 elsewhere, we have, for $\mathbf{Z}$

$$
\Delta=(a, b)^{2} \cup(-b,-a)^{2} \cup(a, b) \times(-b,-a) \cup(-b,-a) \times(a, b) .
$$

We have a generalization of two-bands stationary processes of Section 1.2 (or baseband when $a=0$ ).

3) Stationary processes $\mathbf{Z}$ are harmonizable processes. In this case, $S_{Z}(u, v)$ can be viewed as masses distributed on the bisector $u=v$. Cyclostationary processes, another interesting category of harmonizable processes verify

$$
\mathrm{E}\left[Z(t) Z^{*}\left(t^{\prime}\right)\right]=\mathrm{E}\left[Z(t+T) Z^{*}\left(t^{\prime}+T\right)\right]
$$


for some $T>0 . S_{Z}(u, v)$ is distributed along the set of lines $u=v+k T, k \in \mathbb{Z}$ [22].

For the harmonizable process $\mathbf{Z}$, we can expect that an isometry links $Z(t)$ to $e^{i \omega t}$, taking into account that the second member of (35) defines a scalar product $\left\langle e^{i . t}, e^{i . t^{\prime}}\right\rangle$ (see Appendix B and [21]). The convergence of $g_{N}(., t)$ towards $g(., t)$ in the space matched with $S_{Z}(u, v)$ ) is equivalent to ( $\Delta$ is two-dimensional)

$$
\lim _{N \rightarrow \infty} \iint_{\Delta}\left(g(u, t)-g_{N}(u, t)\right)\left(g(v, t)-g_{N}(v, t)\right)^{*} d S_{Z}(u, v)=0
$$

to compare with $(\Delta$ is one-dimensional)

$$
\lim _{N \rightarrow \infty} \int_{\Delta}\left|g(\omega, t)-g_{N}(\omega, t)\right|^{2} s_{Z}(\omega) d \omega=0 .
$$

Convergence criteria are not very different. This means that it is reasonable to think that formulas of Sections 1 or 2 work for many harmonizable processes (for well-chosen $\Delta$ ).

\section{Conclusion}

This paper addresses properties of PNSN (Periodic Nonuniform Sampling of order $N$ ) applied to stationary processes. Numerous papers have been written in this framework up to today ([23], [24], [25], [26], [27] for instance). Here, sampling subsequences $\mathbf{t}_{k}=\left\{n T+\theta_{k}, n \in \mathbb{Z}\right\}, k=1, . ., N$, are fitted to power spectra inside the union of $N$ intervals $\delta_{j}$ of width $2 \pi / T$. The sampling plan verifies the Landau condition (see Sections 1.2 and 1.3) for errorless reconstruction provided that the set of $\theta_{k}$ is well-chosen. Using the usual sampling formula, an errorless reconstruction is deduced, which is based on the inversion of a $N \times N$ matrix only depending on the delays $\theta_{k}$. Computations provide straight components on intervals $\delta_{j}$, without resampling, that is without taking an intermediary research of values at times $n T / N, n \in \mathbb{Z}$, for instance, and without taking into account possible aliasings.

Results are applied to simple power spectral supports, baseband or two-bands. They allow to obtain straightforwardly the analytic signal, and we explain why appropriate linear filterings allow to improve convergence rates. In some circumstances, as the study of ice cores, the method allows an accurate estimation of power spectra. We explain limitations of the method. When the Landau or the Nyquist condition is not fulfilled, best mean-square estimations can be found. This point is explained for $N=2$ and 3 , and can be generalized at the price of calculus complexity. On the contrary, formulas of reconstruction may be true, suppressing the stationarity hypothesis, provided conditions on two-dimensional spectra.

\section{References}

[1] A. Papoulis, Signal Analysis, Mac-Graw Hill, 1977. 
[2] B. Lacaze, The ghost sampling sequence method, Sampl. Theory Signal Image Process., 8 (1), 13-21, 2009.

[3] B. Lacaze, Reconstruction formula for irregular sampling, Sampl. Theory Signal Image Process., 4 (1), 33-43, 2005.

[4] H. J. Landau, Sampling, data transmission, and the Nyquist rate, Proc. of the IEEE, 35 (10), 1701-1706, 1967.

[5] J. L. Yen, On nonuniform sampling of bandwidth-limited signals, IRE Trans. on Circ. Th. , CT-3 (12), 251-257, 1956.

[6] A. Kohlenberg, Exact interpolation of band-limited functions, J. Applied Physics, 24 (12), 1432-1436, 1953.

[7] J-A. Vernhes et al., Adaptative estimation and compensation of the time delay in a PNS scheme, Proc. SampTA 2015, Washington DC, 2015.

[8] B. Ja. Levin, Distribution of Zeros of Entire Functions, Amer. Math. Soc. Trans. Math. Monographs, Vol. 5, Providence, Rhode Island, 1964.

[9] B. Lacaze, Reconstruction of stationary processes sampled at random times. In Nonuniform Sampling, F. Marvasti (ed.), Kluvers Acacemic, 361-391, 2001.

[10] D. Bonacci and B. Lacaze, Lowpass/bandpass signal reconstruction and digital filtering from nonuniform samples, Proc. ICASSP 2015, Brisbane, 2015.

[11] J. R. Petit, J. Jouzel et all, Climate and atmospheric history of the past 420,000 years from the Vostok ice core, Antartica, Nature 399, 429-436, 1999.

[12] D. Bonacci and B. Lacaze, New $\mathrm{CO}_{2}$ concentration predictions and spectral estimation applied to the Vostok ice core, to appear.

[13] P. Babu and P. Stoica, Spectral analysis of nonuniformly sampled data- a review, Digital Signal Processing, 20 , 359-378, 2010.

[14] M. L. Bender et al., Gas age-ice age differences and the chronology of the Vostok ice core, 0-100 ka, J. of Geophysical Research, 111 , D21, 115, 2006.

[15] K. Kawamura et al., Northern Hemisphere forcing of climatic cycles in Antartica over the past 360,000 years, Nature Letter, 448, 912-916, 2007.

[16] B. Lacaze, Equivalent circuits for the PNS2 sampling scheme, IEEE Trans. on Circuits and Systems-I, 57 (11) , 2904-2914, 2010.

[17] B. Lacaze, Equivalent Circuits for the PNS3 Sampling Scheme, Sampl. Theory Signal Image Process., 12 (2-3), 245-265, 2013.

[18] B. Lacaze, About bi-periodic samplings, Sampl. Theory Signal Image Process., 8(3), 287306, 2009.

[19] M. Loeve, Probability Theory, Van Nostrand, Princeton, 1955.

[20] A. M. Yaglom, Correlation Theory of Stationary and Related Functions, 1, 2, Springer, New-York, 1987.

[21] R. Averkamp, Conditions for the completeness of the spectral domain of a harmonizable process, Stochastic Processes and Their Applications, 72, 1-9, 1997.

[22] W. M. Gardner, A. Napolitano and L. Paura, Cyclostationarity: Half a century of research, Signal Processing, 86 (4) (), 639-697, 2006.

[23] Y. Lin, P. P. Vaidyanathan, Periodic Nonuniform Sampling of Bandpass Signals, IEEE Trans. on Circuits and Systems-II, 45 (3) (3-1998) 340-351.

[24] R. Venkataramani and Y. Bresler, Sampling Theorems for Uniform and Periodic Nonuniform MIMO Sampling of Multiband Signals, IEEE Trans. on Signal Proc., 51 (12) 3152-3163, 2003.

[25] R. S. Prendergrast, B. C. Levy, P. J. Hurst, Reconstruction of band-limited periodic nonuniformly sampled signals through multirate filters banks, IEEE Trans. on Circuits and Systems-I, $\mathbf{5 1}$ (8), 1612-1622, 2004.

[26] S. K. Sindhi, K. M. M. Prabhu, Reconstruction of N-th order nonuniformly sampled bandlimited signals using digital filter banks, Digital Signal Processing, 23, 1877-1886, 2013. 
[27] J. Wang, S. X. Tao, G. H. Chen, Q. X. Peng, Nonuniform optical sampling signal reconstruction algorithm, Optics Comm., 382, 311-317, 2017.

[28] B. Lacaze, A theoretical exposition of stationary processes sampling, Sampl. Theory Signal Image Process., 4 (3) 201-230, 2005.

\section{Appendix A:. Fourier Series Expansion}

The FSE (Fourier Series Expansion) of $e^{i \omega t}$ on $\delta_{k}=\left(a_{k}-\frac{\pi}{T}, a_{k}+\frac{\pi}{T}\right)$ is $(\operatorname{sinc} x=(\sin x) / x)$

$$
\left\{\begin{array}{c}
e^{i \omega t}=\sum_{n \in \mathbb{Z}} c_{n k}(t) e^{i \omega n T} \\
c_{n k}(t)=\frac{T}{2 \pi} \int_{\delta_{k}} e^{i \omega(t-n T)} d \omega=e^{i a_{k}(t-n T)} \operatorname{sinc} \pi\left(\frac{t}{T}-n\right)
\end{array}\right.
$$

which implies, for all $\theta[28]$

$$
\left\{\begin{array}{c}
e^{i \omega t}=\sum_{n \in \mathbb{Z}} c_{n k}(t-\theta) e^{i \omega(n T+\theta)} \\
Z_{k}(t)=\sum_{n \in \mathbb{Z}} c_{n k}(t-\theta) Z_{k}(n T+\theta)
\end{array}\right.
$$

\section{Appendix B:. Linear Invariant Filter (LIF)}

1) The Linear Invariant Filter (LIF) $\mathcal{F}$ links the input $\mathbf{Z}$ to the output $\mathbf{U}=\mathcal{F}[\mathbf{Z}]$ by the formulas (in simple cases)

$$
\left\{\begin{array}{c}
U(t)=\int_{-\infty}^{\infty} f(u) Z(t-u) d u \\
F(\omega)=\int_{-\infty}^{\infty} f(u) e^{-i \omega u} d u
\end{array}\right.
$$

where $f(t)$ and $F(\omega)$ are the impulse response and the complex gain of the LIF. The computations about linear transformations can be made easier using the isometry linking both Hilbert spaces $\mathbf{H}(\mathbf{Z})$ and $\mathbf{L}^{2}\left(s_{Z}\right)[28] . \mathbf{H}(\mathbf{Z})$ is the set of linear combinations of the $Z(t), t \in \mathbb{R}$, where the scalar product $\langle., .\rangle_{\mathbf{H}(\mathbf{Z})}$ is defined by

$$
\langle Z(u), Z(v)\rangle_{\mathbf{H}(\mathbf{Z})}=\mathrm{E}\left[Z(u) Z^{*}(v)\right] .
$$

The induced distance is the classical mean-square convergence. $\mathbf{L}^{2}\left(s_{Z}\right)$ is defined by the set of complex functions, linear combinations of the $e^{i \omega t}, t \in \mathbb{R}$, where the scalar product $\langle., .\rangle_{\mathbf{L}^{2}\left(s_{Z}\right)}$ is defined by

$$
\left\langle e^{i . u}, e^{i . v}\right\rangle_{\mathbf{L}^{2}\left(s_{Z}\right)}=\int_{-\infty}^{\infty} e^{i \omega(u-v)} s_{Z}(\omega) d \omega .
$$

In this framework, the "fundamental isometry" $I_{Z}$ is defined by

$$
Z(t) \longleftrightarrow I_{Z} e^{i . t}
$$

and is generalized to linear combinations so that

$$
\left\{\begin{array}{c}
A \longleftrightarrow I_{Z} f, B \overleftrightarrow{I_{Z}} g \\
\langle f, g\rangle_{\mathbf{L}^{2}\left(s_{Z}\right)}=\langle A, B\rangle_{\mathbf{H}(\mathbf{Z})}
\end{array}\right.
$$

About LIF, we have simultaneously

$$
Z(t) \longleftrightarrow I_{Z} e^{i \omega t}, \mathcal{F}[\mathbf{Z}](t) \longleftrightarrow I_{Z} F(\omega) e^{i \omega t}
$$


As main consequence for sampling, expanding $\mathcal{F}[\mathbf{Z}](t)$ in a linear combination of the data $Z\left(t_{j}\right)$ is equivalent to develop $F(\omega) e^{i \omega t}$ in a linear combination of the $e^{i \omega t_{j}}$.

2) The power spectrum of $\mathbf{Z}_{k}$ cancels outside $\delta_{k}$, which implies that it suffices to expand $F(\omega) e^{i \omega t}$ on $\delta_{k}$ to obtain (21) $\left(e^{i a_{k} n T}=1\right.$ from (19)). Both members of the result are multiplied by $e^{i \omega \theta}$, and $t$ is changed in $t-\theta$. Finally the isometry is used which leads to

$$
\begin{gathered}
F(\omega) e^{i \omega(t+\theta)}=e^{i t a_{k}} \sum_{n=-\infty}^{\infty} f_{n}(t) e^{i \omega(n T+\theta)} \\
\mathcal{F}\left[\boldsymbol{Z}_{k}\right](t) e^{-i(t-\theta) a_{k}}=\sum_{n=-\infty}^{\infty} f_{n}(t-\theta) Z_{k}(n T+\theta)
\end{gathered}
$$

for all $k$. The shape of the second member implies (21). Same operations lead to $(24)$ when (19) is changed in (23). 